\title{
Estradiol and testosterone concentrations in follicular fluid as criteria to discriminate between mature and immature oocytes
}

L.O.B. Costa, M.C. Mendes, R.A. Ferriani, M.D. Moura, R.M. Reis and M.F. Silva de Sá
Setor de Reproducão Humana, Departamento de Ginecologia e Obstetrícia, Faculdade de Medicina de Ribeirão Preto, Universidade de São Paulo, Ribeirão Preto, SP, Brasil

\section{Correspondence}

M.F. Silva de Sá

Departamento de Ginecologia e

Obstetrícia

FMRP, USP

Av. Bandeirantes, 3900

14049-900 Ribeirão Preto, SP

Brasil

E-mail: mfsdsa@fmrp.usp.br

Publication supported by FAPESP.

$\ldots \ldots \ldots \ldots \ldots \ldots$

Received May 17, 2004

Accepted August 3, 2004

\begin{abstract}
The objective of the present study was to examine the association between follicular fluid (FF) steroid concentration and oocyte maturity and fertilization rates. Seventeen infertile patients were submitted to ovulation induction with urinary human follicle-stimulating hormone, human menopausal gonadotropin and human chorionic gonadotropin (hCG). A total of 107 follicles were aspirated after hCG administration, the oocytes were analyzed for maturity and 81 of them were incubated and inseminated in vitro. Progesterone, estradiol (E2), estrone, androstenedione, and testosterone were measured in the FF. E2 and testosterone levels were significantly higher in FF containing immature oocytes (median $=618.2$ and $16 \mathrm{ng} / \mathrm{ml}$, respectively) than in FF containing mature oocytes (median $=368$ and $5.7 \mathrm{ng} / \mathrm{ml}$, respectively; $\mathrm{P}<0.05$ ). Progesterone, androstenedione and estrone levels were not significantly different between mature and immature oocytes. The application of the receiver-operating characteristic curve statistical approach to determine the best cut-off point for the discrimination between mature and immature oocytes indicated levels of 505.8 $\mathrm{ng} / \mathrm{ml}$ for E2 (81.0\% sensitivity and $81.8 \%$ specificity) and of $10.4 \mathrm{ng} /$ $\mathrm{ml}$ for testosterone ( $90.9 \%$ sensitivity and $82.4 \%$ specificity). Follicular diameter was associated negatively with $\mathrm{E} 2$ and testosterone levels in FF. There was a significant increase in progesterone/testosterone, progesterone/E2 and $\mathrm{E} 2 /$ testosterone ratios in $\mathrm{FF}$ containing mature oocytes, suggesting a reduction in conversion of $\mathrm{C} 21$ to $\mathrm{C} 19$, but not in aromatase activity. The overall fertility rate was $61 \%$ but there was no correlation between the steroid levels or their ratios and the fertilization rates. E2 and testosterone levels in FF may be used as a predictive parameter of oocyte maturity, but not for the in vitro fertilization rate.
\end{abstract}

\section{Introduction}

The steroids synthesized by follicular cells during the process of follicle maturation accumulate in follicular fluid (FF). Since the
Key words - Steroid in follicular fluid

- Maturity of oocytes

- In vitro fertilization corona-cumulus-oocyte complex is in close contact with FF, an association is believed to exist between the hormonal content of FF and the quality and degree of oocyte maturity, and therefore FF hormonal content is 
presumed to be related to fertilization and embryo development and implantation rate $(1,2)$.

Gonadotropin-releasing hormone ( $\mathrm{GnRH})$ agonists have been routinely used over the last decade as adjuvants in ovulation induction. These drugs reduce steroidogenesis both in vivo and in vitro in granulosa cells and reduce steroid levels in FF (3). On the other hand, the use of GnRH agonists in hyperinduced cycles has considerably improved the results of assisted fertilization, causing the association between steroid levels and oocyte maturity to be questioned.

Some investigators have tried to determine whether progesterone and estradiol levels in FF or their ratio may be used as parameters to discriminate between ovulatory (mature) oocytes and non-ovulatory (immature) oocytes, since progesterone, estradiol and testosterone levels in FF are significantly modified during the preovulatory period, especially after the luteinizing hormone ( $\mathrm{LH})$ surge (4). However, the results were contradictory $(5,6)$.

In order to improve the techniques of assisted fertilization and to combine better results with reduced costs, some investigators have suggested oocyte retrieval without ovarian hyperstimulation. Immature retrieved oocytes are submitted to in vitro maturation followed by insemination, with a considerable reduction of labor and of operational and medication costs $(7,8)$. Thus, it is important to obtain detailed information about the oocyte environment, especially the composition and influence of FF on the process of oocyte maturation, since the culture media could be improved by adding exogenous steroids.

The objectives of the present study were to determine the levels of steroids in FF, the relationship between them and the degree of oocyte maturation and the rate of in vitro fertilization, in order to establish cut-off values for steroid levels that will differentiate between mature and immature oocytes.

\section{Patients and Methods}

Seventeen infertile couples seen at the Human Reproduction Outpatient Clinic of the Department of Gynecology and Obstetrics, Hospital das Clínicas, Faculty of Medicine of Ribeirão Preto, University of São Paulo, were studied. The couples had been previously selected for assisted fertilization according to a protocol established by the sector. Patient age ranged from 24 to 40 years (mean, 30.6 years). The Research Ethics Committee of the Hospital approved the study and all couples gave written informed consent to participate.

The patients were initially submitted to hyperinduction of ovulation with 2 ampoules of urinary human follicle-stimulating hormone (75 IU/amp; Serono, São Paulo, SP, Brazil) and 2 ampoules of human menopausal gonadotropin (hMG, 75 IU/amp; Ferring, São Paulo, SP, Brazil) at 16:00 h on days 3 and 4 . Starting on the 5th day, stimulation was continued with $\mathrm{hMG}$ alone, 2 ampoules a day at 16:00 $\mathrm{h}$. From the 6th day on, the hMG dose varied from 3 to 4 ampoules according to ovarian response, as evaluated by daily determination of plasma estradiol levels and by daily ultrasound examination up to the day of retrieval included. If estradiol levels increased, preferably doubling in value, the 2 ampoules per day were maintained; if not, the hMG dose was increased up to 4 ampoules per day until the day of human chorionic gonadotrophin (hCG; Serono) administration with daily reevaluations.

When plasma estradiol levels reached $300 \mathrm{pg} / \mathrm{ml}$ per follicle of $12 \mathrm{~mm}$ or more and/ or 2 or more follicles measuring more than $17 \mathrm{~mm}$ in diameter were observed, 10,000 IU hCG were administered up to $52 \mathrm{~h}$ after the last hMG dose. A fall in estradiol levels of at least $50 \%$ and/or ultrasonographic signs of follicular rupture were considered to be criteria for cycle cancellation.

Follicle aspiration for oocyte retrieval 
was performed 34 to $36 \mathrm{~h}$ after hCG administration between 8:00 and 9:00 am. One hundred and seven oocytes and FF were retrieved by follicle aspiration by the transvaginal route under ultrasound guidance. After ovary identification, the number of follicles was determined and follicular diameter was determined by direct measurement before follicle aspiration.

The whole FF was aspirated until the follicle walls collapsed. Only FF free of blood was used to determine hormone concentrations. For FF selection we used the visual criterion proposed by Levay et al. (9). The FF volume obtained after aspiration was measured and the FF was immediately poured onto Petri dishes previously heated to $37^{\circ} \mathrm{C}$ on a hot plate. The content was examined under the light microscope at 80X magnification in order to identify the corona-cumulus-oocyte complex and after the presence of the latter was confirmed, previously identified FF was collected into Falcon tubes and then centrifuged at 2,500 $\mathrm{rpm}$. The supernatant was separated and stored at $-20^{\circ} \mathrm{C}$ until the hormone measurements.

The corona-cumulus-oocyte complexes were first identified under a stereomicroscope and then evaluated for maturity under an inverted photomicroscope and the oocytes were classified as immature, intermediate and mature according to the criteria of Testart et al. (10) and Veeck et al. (11). Of the total of 107 oocytes retrieved, 32 of them were mature, 37 were intermediate and 38 were immature. Eighty-one oocytes were incubated and inseminated according to the in vitro fertilization techniques used in our laboratory. After 18-22 h of insemination, we determined whether or not fertilization had occurred. The criteria were visualization of the second polar corpuscle, the appearance of two pronuclei, and eventually cell division. The oocytes classified as immature were incubated for $24 \mathrm{~h}$ before insemination.

Estradiol, progesterone, testosterone, es- trone, and androstenedione were determined by radioimmunoassay. The FF samples were diluted to varying extents according to the hormone to be measured. Thus, before the assay of each steroid an FF sample was submitted to successive dilutions and the results obtained were used to construct a curve that was compared to the standard curve for the assay, indicating the ideal dilution for the steroid under study, which was the point closest to the $\mathrm{ED}_{50}$ of the standard curve. Two curves were constructed for each steroid, one obtained by measurement with extraction and the other without extraction. Both curves were always parallel to the standard curve after successive dilutions, a fact demonstrating that previous extraction was not necessary for the determination of these hormones. The determinations were carried out in duplicate and all samples from the same subject were analyzed in the same assay. All hormonal assays showed interassay errors of less than $15 \%$ and intra-assay errors below $17 \%$.

\section{Statistical analysis}

Data were analyzed statistically by the chi-square test, Kruskal-Wallis and MannWhitney tests and by Spearman correlation. The level of significance was set at $\mathrm{P}<0.05$ in all tests.

To determine the steroid levels in FF that might discriminate between mature and immature follicles (best relation between sensitivity and specificity) we applied the receiver-operating characteristic (ROC) curve (12). For these calculations we did not consider oocytes classified as intermediate and the "gold standard" was microscopic analysis of the oocytes as described previously $(10,11)$.

\section{Results}

The steroid levels detected in FF are presented in Table 1 according to degree of 
maturity. Estradiol levels were significantly lower in the follicles containing mature oocytes than in the follicles containing immature oocytes $(\mathrm{P}<0.05$; Figure $1 \mathrm{~A})$. For testosterone (Figure 1B), the follicles of mature oocytes also showed significantly lower concentrations than the follicles with immature oocytes. In contrast, progesterone did not differ between the follicles with mature oocytes and the follicles with immature oo- cytes (Figure 1C).

When the ROC curve was applied to locate the cut-off level for steroids that identify a mature oocyte, estradiol levels of $\leq 505.8$ $\mathrm{ng} / \mathrm{ml}$ were found to have $81.0 \%$ sensitivity and $81.8 \%$ specificity, with an $81.8 \%$ positive predictive value and an $85.7 \%$ negative predictive value (Figure 2A). For testosterone, the ROC curve indicated a cut-off level of $\leq 10.4 \mathrm{ng} / \mathrm{ml}$, with $90.9 \%$ sensitivity and

Table 1. Steroid levels in follicular fluid and degree of oocyte maturity.

\begin{tabular}{lccc}
\hline Steroid & \multicolumn{3}{c}{ Oocyte } \\
\cline { 2 - 4 } & Mature $(\mathrm{N}=32)$ & Intermediate $(\mathrm{N}=37)$ & Immature $(\mathrm{N}=38)$ \\
\hline E2 $(\mathrm{ng} / \mathrm{ml})$ & $368.0(104.2-643.6)^{*}$ & $530.4(119.8-957.6)$ & $618.2(198.4-869.3)^{*}$ \\
$\mathrm{P}(\mathrm{ng} / \mathrm{ml})$ & $5,440.0(2,720.0-9,200.0)$ & $5,200.0(1,760.0-27,520.0)$ & $5,120.0(1,600.0-20,800.0)$ \\
$\mathrm{T}(\mathrm{ng} / \mathrm{ml})$ & $5.7(1.0-17.6)^{*}$ & $8.0(4.0-23.2)$ & $16.0(5.6-29.6)^{*}$ \\
$\Delta 4 \mathrm{n}(\mathrm{ng} / \mathrm{ml})$ & $44.0(14.4-200.0)$ & $40.0(6.0-168.0)$ & $46.6(3.6-360.0)$ \\
E1 $(\mathrm{ng} / \mathrm{ml})$ & $1,160.0(480.0-2,560.0)$ & $896.0(128.0-2,400.0)$ & $868.0(120.0-2,140.0)$ \\
$\mathrm{P} / \mathrm{E} 2$ & $15.4(2.8-110.5)^{*}$ & $9.8(2.7-73.2)$ & $7.3(2.0-29.4)^{*}$ \\
$\mathrm{P} / \mathrm{T}$ & $995.2(272.7-4,533.0)^{*}$ & $736.8(173.7-4,000.0)$ & $325.0(54.0-2,363.0)^{*}$ \\
E2/T & $55.9(16.9-383.3)^{*}$ & $59.7(8.3-133.0)$ & $36.6(9.7-138.4)^{*}$ \\
\hline
\end{tabular}

$\mathrm{E} 2$ = estradiol; $\mathrm{P}=$ progesterone; $\mathrm{T}=$ testosterone; $\Delta 4 \mathrm{~A}=$ androstenedione; $\mathrm{E} 1$ = estrone. The numbers in parentheses are the range.

${ }^{*} \mathrm{P}<0.05$ for mature vs immature oocytes (Mann-Whitney and Kruskal-Wallis tests).

Figure 1. Estradiol (E2), progesterone $(\mathrm{P})$, and testosterone $(\mathrm{T})$ levels in follicular fluid (FF) and degree of maturity of oocytes retrieved from patients with a cycle hyperstimulated with gonadotropins E2 (A) and T (B) levels were higher in the follicles containing immature oocytes than in the follicles containing mature oocytes ( ${ }^{*} P<0.05$, Mann-Whitney and KruskalWallis tests). There was no difference in $\mathrm{P}$ levels $(\mathrm{C})$ between oocyte groups $(P>0.05$, MannWhitney test).

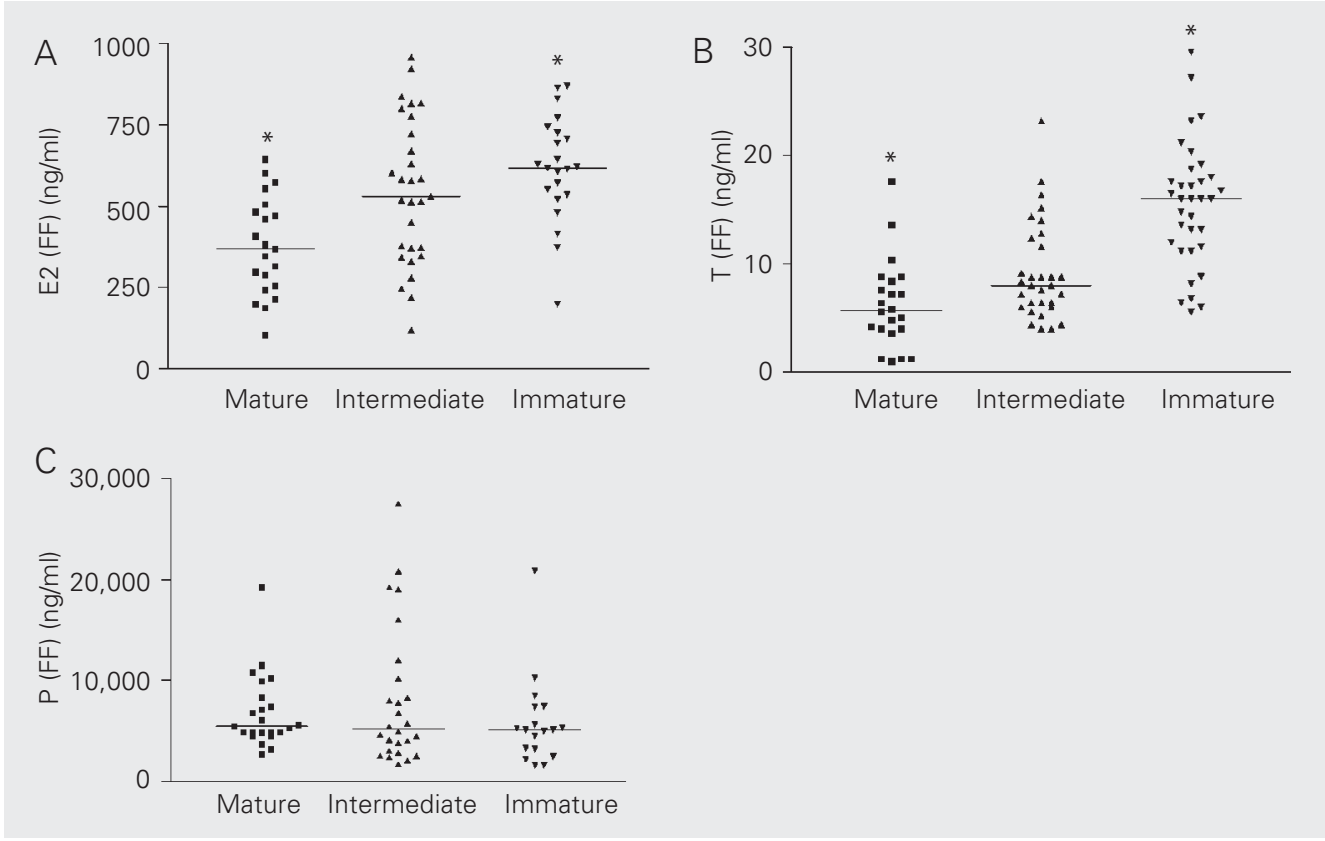


$82.4 \%$ specificity, $76.9 \%$ positive predictive value, and $93.3 \%$ negative predictive value (Figure 2B).

The progesterone/estradiol (Figure 3A), progesterone/testosterone (Figure $3 \mathrm{~B}$ ) and estradiol/testosterone ratios were significantly higher for the follicles with mature oocytes than for the follicles with immature oocytes (Figure 3B, Table 1).

With respect to androstenedione and es- trone, no significant differences were observed between these hormone concentrations in the various follicles.

The levels of steroids in the FF of oocytes of intermediate maturity did not differ significantly from those in the FF of mature or immature oocytes.

When follicular diameter and steroid levels in FF were analyzed, a significant negative correlation was observed between folli-

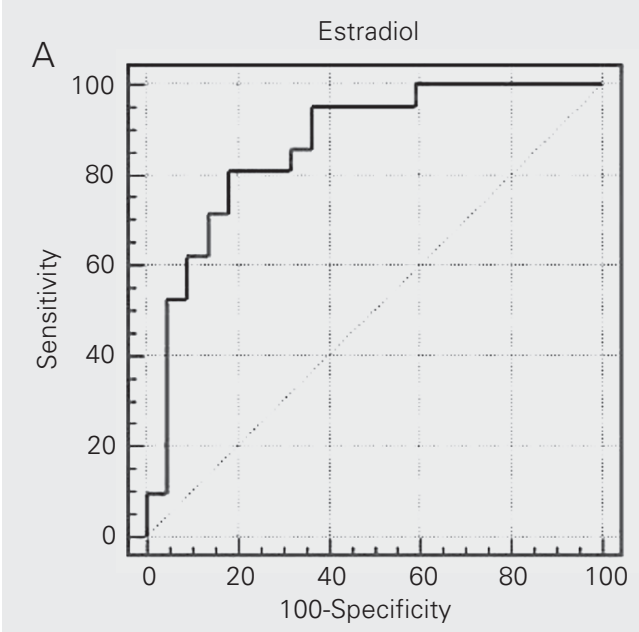

Figure 2. Sensitivity and specificity relation for measurements of estradiol and testosterone in follicular fluid (receiver-operating characteristic curve) as a cutoff point for the identification of mature oocytes. A, Estradiol: cut-off value $=505.8 \mathrm{ng} / \mathrm{ml}$, with $81.0 \%$ sensitivity and $81.8 \%$ specificity. $B$, Testosterone: cutoff value $=10.4 \mathrm{ng} / \mathrm{ml}$, with $90.9 \%$ sensitivity and $82.4 \%$ specificity.
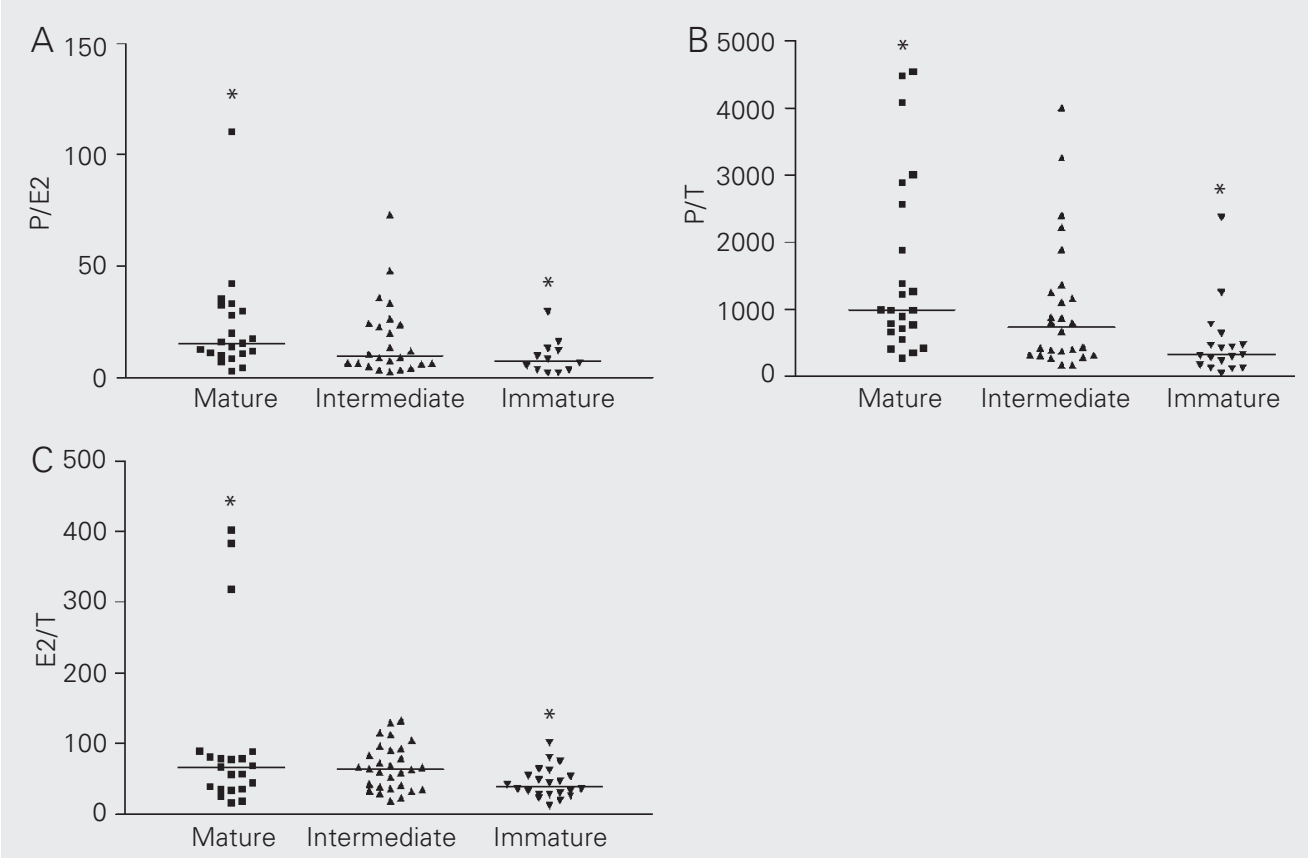

Figure 3. Progesterone/estradio (P/E2), progesterone/testosterone $(\mathrm{P} / \mathrm{T})$ and estradiol/testosterone $(E 2 / T)$ ratio in follicular fluid and degree of maturity of oocytes retrieved from patients with a cycle hyperstimulated with gonadotropins. The P/E2 (A), $P / T(B)$ and $E 2 / T(C)$ ratios were higher in the follicles containing mature oocytes than in the follicles containing immature oocytes $\left({ }^{*} P<0.05\right.$, KruskalWallis and Mann-Whitney tests). 
cular diameter and estradiol levels $(\mathrm{r}=-0.46$; $\mathrm{P}<0.02$ ) and also between follicular diameter and testosterone levels $(\mathrm{r}=-0.5 ; \mathrm{P}<$ 0.005).

No correlation was detected between progesterone, androstenedione and estrone levels in FF and follicular diameter. When estradiol, progesterone and testosterone levels in FF were analyzed for a possible correlation, a significant positive correlation was observed only between estradiol and testosterone levels $(\mathrm{r}=0.39 ; \mathrm{P}<0.01)$.

The overall fertility rate was $61 \%$ and when we compared estradiol, progesterone, and testosterone levels, the progesterone/ estradiol, estradiol/testosterone and progesterone/testosterone ratios and the fertilization rates we did not find differences between fertilized and non-fertilized oocytes.

\section{Discussion}

We analyzed steroid levels in FF and their ratios and related them to the degree of maturity and fertilization rate of oocytes from patients submitted to ovarian stimulation with gonadotrophins only. Since the objective of the present study was to investigate the physiological phenomenon, no GnRH agonist was used because of a possible effect on steroidogenesis $(3,13)$.

It is known that a positive association between estradiol and follicular diameter occurs in plasma during the follicular phase both in natural and induced cycles. For FF, however, Uehara et al. (14) demonstrated a positive association between estradiol and follicular volume only for smaller follicles, an association that disappears when the follicles are larger than $8 \mathrm{ml}$.

Thus, before the LH surge, estradiol levels in the fluid of preovulatory follicles increase progressively in a manner proportional to the activity of the aromatase system in granulosa cells (15). In contrast, a few hours after the onset of the ovulatory LH surge, ovarian estradiol secretion decreases abruptly, and consequently estradiol levels in follicular fluid and plasma are also markedly reduced $(4,16)$.

Mature oocytes are associated with reduced estradiol levels in FF fluid after hCG, as shown by the present results. It seems that the ovulatory LH surge exerts its effect both on this inversion of the steroidogenic pattern and on the process of oocyte maturation (17). Indeed, we observed a negative correlation between follicle diameter and estradiol levels in FF. This decline in estradiol production seems to be mainly due to the inhibition of the synthesis of androgen precursors by thecal cells brought about by desensitization of these cells to LH induced by the LH surge itself, with the cells becoming refractory to this gonadotropic stimulus $(14,18,19)$.

Several investigators have demonstrated that mature oocytes with a higher potential for fertilization are also associated with lower testosterone levels in FF at the time of follicular rupture $(20,21)$. Our results are in agreement with these data. The association between oocyte maturity and low testosterone concentration in FF seems to be expected, since atresia of the follicles and of their oocytes is usually associated with granulosa cell degeneration and with an increased testosterone/estradiol ratio (22).

Progesterone levels were not different between mature and immature oocytes. The maintenance of progesterone production occurring in parallel to the reduction in estradiol and testosterone levels in preovulatory follicles seems to result from a modification of the steroidogenic patterns of granulosa cells. Oocyte maturation is accompanied by a significant reduction in estradiol and testosterone levels with a positive association between them. Indeed, this could not be different, since androgens and testosterone in particular are immediate precursors of estradiol. Thus, if a reduction in testosterone production by thecal cells is observed this fact definitely contributes to the reduction of 
estrogen synthesis observed in parallel.

It appears that the most relevant feature in the process of oocyte maturation is the relation between steroids. Some investigators, using the steroid ratios in FF, suggested that the progesterone/estradiol ratio may be the best indicator of maturity (23) and of the potential for oocyte fertilization (24). We observed that the progesterone/estradiol, progesterone/testosterone and estradiol/testosterone ratios were higher in $\mathrm{FF}$ from follicles of mature oocytes. An increase in the progesterone/testosterone ratio corresponds to a reduction in the metabolism of $\mathrm{C} 21$ (progestogens) to $\mathrm{C} 19$ (androgens). The positive association between estradiol and testosterone and the increase in the estradiol/testosterone ratio suggest that the fall in estradiol levels may be due to the reduction of testosterone rather than to a reduction of aromatase activity.

With respect to androstenedione levels, the results showed no relationship with the degree of oocyte maturity. This absence of an association between androstenedione levels and degree of oocyte maturity may reflect the intermediate role of this steroid as a precursor of estrogen biosynthesis.

It should be pointed out that the hormonal content of the follicles that contained oocytes of intermediate maturity did not have any specific patterns in terms of steroid levels in the FF, as was observed for mature and immature oocytes. Because of the asynchrony in follicular development characteristic of all stimulated cycles, it is possible that the follicles containing intermediate oocytes were in different stages of steroidogenesis at the time of follicle aspiration, with a tendency to show secretion patterns of a mature follicle at times and of an immature follicle at others.

In view of the high sensitivity and specificity indices obtained in the present study for the cut-off points for testosterone and estradiol, we suggest the possibility of using these indices to predict oocyte maturity based on the levels of these steroids in FF. Current techniques for steroid determination permit obtaining rapid results within a period of up to $90 \mathrm{~min}$, a much shorter time compared to the interval between oocyte aspiration and oocyte insemination or sperm injection in cases of intracytoplasmic sperm injection. Thus, from a technical viewpoint, it would be quite possible to use hormonal determinations in FF for the assessment of oocyte maturity and for oocyte selection.

The present data acquire special importance if we consider recent studies using assisted fertilization techniques without stimulating ovulation $(7,8)$. These techniques have been mainly used in "high responder" patients who would be likely to develop the ovarian hyperstimulation syndrome. As an alternative to ovarian hyperstimulation, the cited investigators $(7,8)$ propose the retrieval of immature oocytes obtained from follicles measuring up to $10 \mathrm{~mm}$ in diameter and their later maturation in vitro. Thus, in these situations the time elapsed between oocyte retrieval and insemination could be prolonged. The results reported by these investigators are encouraging, with rates of in vitro maturation above $60 \%$, fertilization rates of $70 \%$ and pregnancy rates of about $30 \%$. The results of these procedures can be improved and one of the factors that will definitely contribute to this improvement will be the development of more appropriate culture media for the oocytes, with the addition of steroids in appropriate proportions. On this basis, information about the physiological processes of oocyte maturation is of fundamental importance. These techniques involve a significant reduction in costs since they require minimum medication expenses, without the need for continuous patient monitoring. This reduction in costs is of high medical and social interest since it may provide access to assisted fertilization procedures to persons with low acquisitive power.

With respect to the relation between steroid levels in FF and fertilization rates, 
Andersen (25) demonstrated that the estradiol/testosterone ratio is higher in follicles associated with fertilization. Previous studies by Pellicer et al. (2) have shwon that, although the steroid levels in FF differ significantly between follicles of women with and without endometriosis, there is no way to predict the differences in embryo quality between the two groups. In contrast, the values for progesterone and the estradiol/ testosterone ratio were higher in oocytes with poorer prognosis (endometriosis). Our results did not show any differences be- tween fertilized and non-fertilized oocytes for any of the parameters analyzed.

Steroid levels in FF and their ratios differ between mature and immature oocytes. Our results suggest that their measurements, particularly of estradiol and testosterone, may be used as predictive indicators of oocyte maturity since they presented high sensitivity, specificity and predictive values. However, they cannot be used as parameters for the prediction of whether an oocyte will have a higher or lower probability of being fertilized in vitro.

\section{References}

1. Fishel SB, Edwards RG \& Walters DE (1983). Follicular steroid as a prognosticator of successful fertilization of human oocytes in vitro. Journal of Endocrinology, 99: 335-344.

2. Pellicer A, Valbuena D, Bauset C, Albert C, Bonilla-Musoles F, Remohi J \& Simon C (1998). The follicular endocrine environment in stimulated cycles of women with endometriosis: steroid levels and embryo quality. Fertility and Sterility, 69: 1135-1141.

3. Dor J, Bider D, Shulman A, Levron JL, Shine S, Mashiach S \& Rabinovici J (2000). Effects of gonadotrophin-releasing hormone agonists on human ovarian steroid secretion in vivo and in vitro. Results of a prospective, randomized in vitro fertilization study. Human Reproduction, 15: 1225-1230.

4. Murdoch WJ \& Dunn TG (1982). Alterations in follicular steroid hormones during the preovulatory period in the ewe. Biology of Reproduction, 27: 300-307.

5. Peek JC, Ghoy VJ, Watkins WB \& Graham FM (1986). Levels of oxytocin-like activity and progesterone in follicular fluid from in vitro fertilization cycles. Journal of In Vitro Fertilization and Embryo Transfer, 3: 157-165.

6. Wramsby H, Kullander S, Liedholm P, Rannevik G, Sundstrom P \& Thorell J (1981). The success rate of in vitro fertilization of human oocytes in relation to the concentrations of different hormones in follicular fluid and peripheral plasma. Fertility and Sterility, 36: 448454.

7. Cha KY, Han SY, Chung HM, Doi DH, Lim JM, Lee WS, Ko JJ \& Yoon TK (2000). Pregnancies and deliveries after in vitro maturation culture followed by in vitro fertilization and embryo transfer without stimulation in women with polycystic ovary syndrome. Fertility and Sterility, 73: 978-983.

8. Chian RC, Buckett WM, Tulandi T \& Tan SL (2000). Prospective randomized study of human chorionic gonadotrophin priming before immature oocyte retrieval from unstimulated women with polycystic ovarian syndrome. Human Reproduction, 15: 165-170.

9. Levay PF, Huyser C, Fourie FL \& Rossouw DJ (1997). The detection of blood contamination in human follicular fluid. Journal of Assisted Reproduction and Genetics, 14: 212-217.

10. Testart J, Frydman R, De Mouzon J, Lassalle B \& Belaisch JC (1983). A study of factors affecting the success of human fertiliza- tion in vitro. I. Influence of ovarian stimulation upon the number and condition of oocytes collected. Biology of Reproduction, 28: 415424.

11. Veeck LL, Wortham Jr JW, Witmyer J, Sandow BA, Acosta AA, Garcia JE, Jones GS \& Jones Jr HW (1983). Maturation and fertilization of morphologically immature human oocytes in a program of in vitro fertilization. Fertility and Sterility, 39: 594-602.

12. Zweig $\mathrm{MH}$ \& Campbell G (1993). Receiver-operating characteristic (ROC) plots: a fundamental evaluation tool in clinical medicine. Clinical Chemistry, 39: 561-577.

13. Sridaran R, Lee MA, Haynes L, Srivastava RK, Ghose M, Sridaran G \& Smith CJ (1999). GnRH action on luteal steroidogenesis during pregnancy. Steroids, 64: 618-623.

14. Uehara S, Naganuma T, Tsuiki A, Kyono K, Hoshiai H \& Suzuki M (1985). Relationship between follicular fluid steroid concentrations and in vitro fertilization. Obstetrics and Gynecology, 66: 19-23.

15. Hillier SG, Reichert Jr LE \& Van Hall EV (1981). Control of preovulation follicular estrogen biosynthesis in the human ovary. Journal of Clinical Endocrinology and Metabolism, 52: 847-856.

16. Hillier SG, Wickings EJ, Afnan M, Margara RA \& Winston RM (1984). Granulosa cell steroidogenesis before in vitro fertilization. Biology of Reproduction, 31: 679-686

17. Moor RM, Polge C \& Willadsen SM (1980). Effect of follicular steroids on the maturation and fertilization of mammalian oocytes. Journal of Embryology and Experimental Morphology, 56: 319-335.

18. Leung PC \& Armstrong DT (1980). Interactions of steroids and gonadotropins in the control of steroidogenesis in the ovarian follicle. Annual Review of Physiology, 42: 71-82.

19. Magoffin DA \& Erickson GF (1982). Direct inhibitory effect of estrogen on LH-stimulated androgen synthesis by ovarian cells cultured in defined medium. Molecular and Cellular Endocrinology, 28: 8189.

20. Seibel MM, Smith D, Dlugi AM \& Levesque L (1989). Periovulatory follicular fluid hormone levels in spontaneous human cycles. Journal of Clinical Endocrinology and Metabolism, 68: 1073-1077.

21. Vanluchene $E$, Hinting $A$, Dhont $M$, De Sutter $P$, Van Maele G \& Vandekerckhove D (1991). Follicular fluid steroid levels in relation to oocyte maturity and in vitro fertilization. Journal of Steroid Biochem- 
istry and Molecular Biology, 38: 83-87.

22. Brailly S, Gougeon A, Milgrom E, Bomsel-Helmreich O \& Papiernik E (1981). Androgens and progestins in the human ovarian follicles: differences in the evolution of preovulation, healthy nonovulatory, and atretic follicles. Journal of Clinical Endocrinology and Metabolism, 53: 128-134.

23. Frederick JL, Francis MM, Macaso TM, Lobo RA, Sauer MV \& Paulson RJ (1991). Preovulatory follicular fluid steroid levels in stimulated and unstimulated cycles triggered with human chorionic gonadotropin. Fertility and Sterility, 55: 44-47.

24. Basuray R, Rawlins RG, Radwanska E, Hening I, Sachdeva S, Tummon I, Binor Z \& Dmowski WP (1988). High progesterone/ estradiol ratio in follicular fluid at oocyte aspiration for in vitro fertilization as a predictor of possible pregnancy. Fertility and Sterility, 49: 1007-1111.

25. Andersen CY (1993). Characteristics of human follicular fluid associated with successful conception after in vitro fertilization. Journal of Clinical Endocrinology and Metabolism, 77: 1227-1234. 